\title{
Noam Chomsky
}

\section{Alter Wein in neuen Schläuchen: ein bitterer Nachgeschmack}

Gerade als Jane Kelseys erhellende Studie über das »neuseeländische Experiment « erscheinen sollte, veröffentlichte das »Royal Institute of International Affairs « in London die Jubiläumsausgabe zum 75. Jahrgang seiner Zeitschrift International Affairs mit Artikeln zu wichtigen Themen der Zeit. Einer davon ist den »Experimenten « jener Sorte gewidmet, denen sich Neuseeland unterwirft, sowie ihren intellektuellen Wurzeln. Der Autor, Paul Krugman, gehört zu den führenden Vertretern der internationalen und Entwicklungsökonomie. Er trifft fünf zentrale Feststellungen, die in diesem Zusammenhang ausgesprochen sachdienlich sind.

Erstens stellt er fest, daß das Wissen über ökonomische Entwicklung sehr begrenzt ist. Ein großer Teil der Ursachen für wirtschaftliches Wachstum muß dem »Rest «, dem »Maß unseres Nichtwissens« zugeschrieben werden, wie es Robert Solow nannte. In dem am besten untersuchten Fall, den USA, fallen zwei Drittel der Steigerung des Pro-Kopf-Einkommens in diese Kategorie. Auch die asiatischen NICs bieten »keine eindeutigen Lektionen«. Sie folgen »unterschiedlichen und mehrdeutigen« Wegen, die sicherlich nicht dem entsprechen, was von der »gegenwärtigen Orthodoxie zum Schlüssel für Wachstum erklärt wird «. Krugman empfiehlt »Bescheidenheit« angesichts der Grenzen des Verstehens und Vorsicht gegenüber »raschen Verallgemeinerungen «.

Krugmans zweite Feststellung ist, daß politische Intellektuelle und Planer (darunter viele Ökonomen) dennoch ständig solche Verallgemeinerungen anbieten. Zudem liefern sie die theoretischen Grundlagen für jene politischen Strategien, die umgesetzt werden, wenn es die Umstände erlauben.

Drittens ist die »anerkannte Lehre « nicht dingfest zu machen; sie bewegt sich regelmäßig woanders hin, vielleicht sogar zum Gegenteil der letzten Phase - obwohl die Befürworter immer wieder vor Selbstvertrauen strotzen, wenn sie die neue Orthodoxie durchsetzen. 
Viertens stimmt man im Rückblick immer darin überein, daß die politischen Strategien dem »formulierten Ziel nicht dienten« und auf »schlechten Ideen« beruhten.

Schließlich wird gewöhnlich »erklärt, daß die schlechten Ideen gedeihen, weil sie im Interesse von mächtigen Gruppen liegen. Ohne Zweifel, das passiert...« (Krugman 1995).

$\mathrm{Da}$ es passiert, ist ein Allgemeinplatz mindestens schon seit Adam Smith die merkantilistischen Theorien verdammte, die im Interesse der »Kaufleute und Industriellen « entworfen worden waren, den »hauptsächlichen Architekten« der britischen Politik. Sie mobilisierten die Staatsmacht, um sicher zu sein, daß ihre eigenen Interessen "ganz besonders beachtet würden«, wie »leidvoll« auch immer die Folgen für andere sein würden, das englische Volk eingeschlossen. Es passiert nicht nur, es passiert auch mit beeindruckender Folgerichtigkeit. Das gegenwärtige »neuseeländische Experiment« dringt nicht auf neues Gelände vor, wenn »der Nutzen [der politischen Strategien] rasch dem Unternehmenssektor zufließt«, der ja »offenkundig ... strategischen Einfluß« auf ihre Konzeption hatte, und wenn die spolitischen Akteure die Karten zugunsten von Wählerkreisen mischen, die als Nutznießer vorgesehen sind « (Kelsey 1995: 72f).

Das ist der Kern der Sache, der meiner Ansicht nach eine Reformulierung der Krugmanschen Schlußfolgerungen verlangt. Die »schlechten Ideen« mögen nicht dem »erklärten Ziel « entsprechen, aber sie stellen sich in ganz typischer Weise als sehr gute Ideen für ihre Befürworter heraus. Es gab in der neueren Zeit nicht wenige Experimente zur Wirtschaftsentwicklung, und obwohl es zweifellos klug ist, sich vor raschen Verallgemeinerungen zu hüten, weisen sie trotzdem einige Regelmäßigkeiten auf, die schwer zu übersehen sind. Eine besteht darin, daß die Konstrukteure des Experiments ganz gut aus der Sache herauszukommen scheinen, obwohl die Versuchspersonen, die selten Einwilligungsformulare unterschrieben haben, oft genug Prügel beziehen.

Das erste derartige Experiment fand kurze Zeit nach Smiths Äußerungen statt, im Jahr 1793, als die britischen Herrscher in Indien die »dauerhafte Besiedlung« einführten, die wundersame Dinge bewerkstelligen sollte. 40 Jahre später überprüfte eine britischen Untersuchungskommission die Ergebnisse. Sie kam zu dem Schluß, daß sdie Besiedlung, die mit großer Sorgfalt und Überlegung ausgeführt worden war, wie uns nun schmerzlich bewußt ist, fast die Gesamtheit der unteren Schichten schwerster Unterdrückung ausgeliefert hat« und ein »Elend « hinterläßt, das »in der Geschichte des Handels beispiellos ist«, wie der Direktor der ehrenwerten Gesellschaft hinzufügte, während »die Knochen der Baumwollweber die Ebenen Indiens bleichen «. 
Doch das Experiment kann kaum als Fehlschlag abgeschrieben werden. Generalgouverneur Lord Bentinck bemerkte, daß »die 'dauerhafte Besiedlung', obwohl in anderer Hinsicht und in vielen wichtigen Punkten gescheitert, zum mindesten den großen Vorteil hat, daß sie eine große Anzahl von reichen Landeigentümern schuf, die großes Interesse an der Fortdauer der Britischen Herrschaft und die vollständige Kontrolle über die Masse der Bevölkerung haben «, deren wachsendes Elend deshalb ein geringeres Problem ist, als es hätte sein können. Auch die britischen Investoren sind nicht schlecht weggekommen. Abgesehen vom enormen Reichtum, der Individuen und Unternehmen zuflo $\beta$, finanzierte Indien bald $40 \%$ des britischen Handelsdefizits, während es auf der anderen Seite ein protektionierter Markt für britische Produkte war; Kontraktarbeiter für britische Besitzungen von der Karibik bis nach Afrika, Ceylon und Malaysia »ersetzten die früheren Sklaven«, bemerkt die Cambridge Economic History of India; es lieferte Truppen für Großbritanniens Kolonialkriege und seine europäischen Kriege; und das Opium war der Hauptartikel der britischen Exporte nach China - nicht ganz auf der Grundlage einer freien Marktwirtschaft, deren geheiligte Prinzipien wurden denn auch ignoriert, als diese nützliche Substanz von England ferngehalten wurde (vgl. dazu genauer Chomsky 1993; Bayly 1988).

Kurz gesagt war das erste große Experiment eine »schlechte Idee« für die Untertanen, aber nicht für seine Erfinder und die lokalen Eliten, die mit ihnen verbunden waren. Diese geschickte Fügung hat es mit erstaunlicher Regelmäßigkeit bis zum heutigen Tag immer wieder gegeben. Die Folgerichtigkeit ist nicht weniger beeindruckend als die Höhenflüge der Rhetorik, mit der das jüngste »Schaufenster für Demokratie und Kapitalismus« und das "Versuchsgelände für wissenschaftliche Entwicklungsmethoden« als ein erstaunliches »Wirtschaftswunder« bejubelt wurde -- und gleichermaßen folgerichtig ist, was diese Rhetorik verdeckt.

Das jüngste Beispiel ist Mexiko. Es wurde hoch gelobt für seine strikte Einhaltung der Regeln des »Washingtoner Konsenses«, der auch das Denken der neuseeländischen Technokraten leitet und stolz anderen als Modell angepriesen wurde, während die Löhne in den Keller sanken, die Armutsrate fast genauso schnell anstieg wie die Zahl der Milliardäre, fremdes Kapital einströmte (meist spekulatives oder für die Ausbeutung superbilliger Arbeit bestimmt, die von der brutalen »Demokratie « unter Kontrolle gehalten wurde) und all die anderen Begleiterscheinungen der "Schaufenster « und "Wunder « auftraten. Ebenfalls bekannt ist der Ausgang der Geschichte, der Zusammenbruch des Kartenhauses im Dezember 1994, wie es von Beobachtern vorhergesagt worden war, die es vorzogen, das Geschehen nicht durch das verzerrende Prisma der »schlechten Ideen « zu verfolgen, die »prächtig gedeihen, weil sie im Interesse mächtiger Gruppen liegen«. 
Die Geschichte lehrt auch noch mehr. Im 18. Jahrhundert waren die Unterschiede zwischen der Ersten und der Dritten Welt weit geringer als heute. Zwei Fragen drängen sich daher auf: (1) Welche Länder entwickelten sich und welche nicht? (2) Können wir einige der bestimmenden Faktoren ausfindig machen?

Die Antwort auf die erste Frage ist ziemlich eindeutig. Außerhalb Westeuropas entwickelten sich zwei Regionen: die USA und Japan - das heißt, die beiden Regionen, die in der Lage waren, sich der europäischen Kolonialisierung zu entziehen. Japans Kolonien sind ein Sonderfall, insbesondere weil Japan trotz brutaler Herrschaft seine Kolonien nicht ausbeutete, sondern sie etwa in demselben Ausmaß wie das Mutterland selbst entwickelte.

Wie erging es Osteuropa? Im 15. Jahrhundert begann Europa sich zu teilen, der Westen entwickelte sich und der Osten wurde eine Art Zulieferer, die ursprüngliche Dritte Welt. Die Teilung vertiefte sich zu Anfang dieses Jahrhunderts, dann befreite sich Rußland von diesem System. Trotz der fürchterlichen Stalinschen Greuel und trotz der riesigen Zerstörungen durch die beiden Weltkriege machte die UdSSR, genau wie ihre Satelliten, eine bedeutende Industrialisierung durch. Das ist die »Zweite Welt«, nicht ein Teil der »Dritten Welt « - oder besser, sie war es bis 1989. Bis in die frühen sechziger Jahre hinein, so enthüllen die Dokumente, befürchteten westliche Planer, daß das wirtschaftliche Wachstum den Osten mit dem Westen gleichziehen lassen könnte, und daß der »Vorzeige-Effekt« andere dazu bringen würde, einen Kurs des »wirtschaftlichen Nationalismus« einzuschlagen. Nach dem Ende des Kalten Krieges kehrte der größte Teil Osteuropas zum Status quo ante zurück: Die Regionen, die schon vorher Teil des industrialisierten Westens waren, gewannen diesen Status zurück, während in den übrigen Gebieten wieder Dritte-Welt-Strukturen entstehen.

Die Welt ist komplizierter als irgendeine einfache Beschreibung, aber dies ist eine ziemlich brauchbare erste Annäherung, die uns einiges über das hier behandelte Thema sagt, ebenso wie zum Kalten Krieg. Was sie vermuten läßt, wird auch durch die Beobachtung gestützt, daß, obwohl John F. Kennedys »monolithische und rücksichtslose Verschwörung« zur Eroberung der Welt inzwischen fast vergessen ist, das Budget des Pentagon trotzdem auf dem Niveau des Kalten Krieges geblieben ist und jetzt sogar wieder wächst. Diese Tatsachen führen einen rationalen Menschen zu einigen Schlüssen über die Rolle der sowjetischen Bedrohung in den Überlegungen der Planer; und Washingtons internationale Politik hat höchstens taktische und rhetorische Änderungen erfahren, nun da die Vorwände der Vergangenheit nicht länger bei Bedarf aus dem Regal geholt werden können und mehr Informationen zu Verfügung stehen, die einem das Verständnis des Kalten Krieges ermöglichen. 
Zurück zu Frage (1): Es scheint, daß Entwicklung immer dann stattfand, wenn es keine »Experimente« gab, die auf »schlechten Ideen « beruhten, die freilich gute Ideen für die Planer und ihre Kollaborateure waren. Die Fähigkeit, solche Maßnahmen abzuwehren, garantiert keine ökonomische Entwicklung, aber sie scheint eine Voraussetzung dafür gewesen zu sein.

Wenden wir uns Frage (2) zu. Wie gelang es Europa und jenen Ländern, die sich seinem Zugriff entziehen konnten, sich zu entwickeln? Ein Teil der Antwort scheint ausnahmslos gültig zu sein: durch radikale Verletzung der anerkannten Lehre von der freien Marktwirtschaft. Diese Folgerung gilt von England bis zur ostasiatischen Wachstumsregion von heute, sicher unter Einschluß der USA, »dem Mutterland und der Bastion des modernen Protektionismus«, wie der Wirtschaftshistoriker Paul Bairoch in seiner kürzlichen Studie über die Mythen der ökonomischen Entwicklung bemerkt. Am seltsamsten, schließt er, sei der Glaube, daß Protektionismus wirtschaftliches Wachstum behindere: »Es ist schwierig, einen anderen Fall zu finden, bei dem die Tatsachen derart einer vorherrschenden Theorie widersprechen «; eine Folgerung, die von vielen anderen Studien geteilt wird (Bairoch 1993; Eckes 1995).

Bei der kritischen Bilanz ihrer wirtschaftlichen Entwicklungsprogramme nach dem Zweiten Weltkrieg wies eine Gruppe prominenenter japanischer Ökonomen darauf hin, daß sie sich den neoklassischen Ratschlägen ihrer Berater widersetzten und statt dessen, wie der Herausgeber bemerkte, eine Form der Industriepolitik wählten, die dem Staat eine beherrschende Rolle zuschrieb; ein System, das »eher der Organisation der industriellen Bürokratie in den sozialistischen Ländern gleichkam und keine direkte Entsprechung in irgendeinem fortgeschrittenen westlichen Land hatte (Ryutaro Komiya, Wirtschaftswissenschaftler an der Universität Tokio). »Die 'Ideologie' der Industriepolitik während dieser (frühen Nachkriegs-) Zeit stützte sich nicht auf neoklassische Ökonomie oder keynesianisches Denken, sondern war neomerkantilistischen Ursprungs«, ergänzt ein Mitarbeiter, und war »auch deutlich vom Marxismus beeinflußt«. Marktmechanismen wurden stufenweise von der Staatsbürokratie eingeführt, und Konglomerate aus Industrie- und Finanzunternehmen gewannen als Garanten für wirtschaftlichen Erfolg an Bedeutung. Die Abwehr orthodoxer ökonomischer Prinzipien war eine Bedingung für das japanische Wunder, folgern die Wirtschaftswissenschaftler.

Um zu den früheren japanischen Kolonien zu kommen: Die erste ausführliche Studie der US-Hilfsmission in Taiwan entdeckte, daß die US-Berater und die chinesischen Planer, nobwohl in anglo-amerikanischer Wirtschaftstheorie versiert «, die Lehren und Anordnungen aus Washington mißachteten. Der technische Experte der USA in Taiwan zog es vor, »die Patentrezepte der freien Marktwirtschaft von Anfang an über Bord zu wer- 
fen, mit den chinesischen Beamten zusammenzuarbeiten« und eine »staatszentrierte Strategie zu entwickeln «, während in Taiwan die in der Kolonialzeit begonnene Entwicklung wieder in Gang kam. Die Politik gründete auf dem noch immer gültigen Prinzip »der aktiven Teilnahme der Regierung an den wirtschaftlichen Aktivitäten der Insel mit Hilfe von gut durchdachten Plänen und der Überwachung ihrer Ausführung« (Win 1953). Inzwischen »preisen« die USA »Taiwan als eine Erfolgsgeschichte des freien Unternehmertums an «, genauso wie es die Weltbank heute mit wachsender Verzweiflung tut, während Analytiker, die mit den Tatsachen befaßt sind, die entscheidende und fortdauernde Funktion des »unternehmerischen Staats « beschreiben, der anders als in Südkorea funktioniert, aber unter einer genauso starken Führung (Komya et al. 1988; Cullaher 1996; Weiching Wang 1995-96).

Die zentrale Rolle staatlichen Managements und staatlicher Initiative in Volkswirtschaften, die sich spät entwickelten, ist seit der Arbeit von Alexander Gerschenkron gut bekannt; es muß nur noch hinzugefügt werden, daß Ähnliches seit den ersten Anfängen der industriellen Revolution zutrifft. In diesem Bereich scheinen allerdings nur wenige Aussagen empirisch abgesichert zu sein.

Es ist eine untergeordnete Frage, wie die Dritte Welt das wurde, was sie heute ist. Bairoch gibt eine plausible, wenn auch partielle Antwort: »Es gibt keinen Zweifel, daß der im 19. Jahrhundert der Dritten Welt aufgezwungene Wirtschaftsliberalismus ein wichtiger Grund für die Verzögerung ihrer Industrialisierung ist«, und im dramatischen und sehr aufschlußreichen Fall Indien ist es der »Prozeß der Ent-Industrialisierung «, der die industrielle Werkstatt und das Handelszentrum der Welt in eine zutiefst verarmte Agrargesellschaft verwandelte, verbunden mit einem starken Absinken der Reallöhne, des Nahrungsmittelverbrauchs und der Verfügbarkeit anderer einfacher Erzeugnisse des 18. Jahrhunderts; ein »Unglück, das ohne Beispiel in der Weltwirtschaftsgeschichte ist $\ll$, schließt die detaillierteste moderne Studie (Mukerjee 1967; Rothermund 1993; Chandra 1971).

»Indien war nur das erste große Opfer in einer langen Liste«, so Bairoch, darunter »sogar politisch unabhängige Dritte-Welt-Länder, die gezwungen wurden, ihre Märkte westlichen Produkten zu öffnen«. Inzwischen schützten sich die westlichen Gesellschaften selber vor den Gesetzen des Marktes - mit einer Tendenz zur Einmischung in die Märkte, die man nicht so ohne weiteres außer acht lassen kann, wie Bairoch und andere anmerken.

Wenn man die Details beiseite läßt, so scheint es ziemlich klar, daß ein Grund für die scharfe Teilung zwischen Erster und Dritter Welt darin besteht, daß große Teile der letzteren Gegenstand von "Experimenten« waren, die ihnen die Lehre der freien Marktwirtschaft eintrichterten, während die heute entwickelten Länder solche Maßnahmen abwehren konnten. 
Das bringt uns zu einem anderen Wesenszug der modernen Geschichte, den man kaum auslassen kann - in diesem Fall auf der ideologischen Ebene. Die Lehre der freien Marktwirtschaft erscheint in zwei Gestalten. Die erste ist die offizielle Lehre, die von den gebildeten Schichten gelehrt und den Wehrlosen aufgezwungen wird. Die zweite können wir die »real existierende Lehre der freien Marktwirtschaft« nennen: für dich, aber nicht für mich, außer zugunsten eines augenblicklichen Vorteils. Ich brauche den Schutz des fürsorglichen Staates, aber du mußt ohne solchen Schutz Verantwortung lernen. Diejenigen, die in der Position sind, sich entscheiden zu können, nehmen typischerweise die zweite Version der Lehre der freien Marktwirtschaft an; jene, die, wie die Geschichte zeigt, notwendige, wenn auch nicht hinreichende Vorbedingung für Entwicklung ist.

Fahren wir mit der Untersuchung fort, so entdecken wir schnell, daß die Auswirkungen staatlicher Intervention auf die Wirtschaft in Standarderklärungen, die sich sehr stark auf solche speziellen Fälle wie Protektionismus konzentrieren, häufig unterschätzt werden. Dieser Begriff muß viel weiter gefaßt werden. Um einen offensichtlichen Fall auszusuchen: die frühe industrielle Revolution hing von billiger Baumwolle ab. Diese wurde nicht durch die Anbetung des Marktes billig und verfügbar gehalten; sondern durch die Vertreibung oder Auslöschung der indigenen Bevölkerung des amerikanischen Südens in Verbindung mit der Sklaverei. Außerdem gab es damals weitere Baumwollproduzenten. Führend unter ihnen war Indien unter der Kolonialherrschaft, so daß seine Ressourcen nach England flossen, während seine eigene, bedeutend weiter entwickelte Textilindustrie durch die harte und bewußte Anwendung der »real existierenden Lehre der freien Marktwirtschaft« zerstört wurde. Ein anderer Fall ist Ägypten, das zur selben Zeit mit der industriellen Entwicklung begann wie Neuengland, aber durch britische Gewalt von der weiteren Entwicklung ausgeschlossen wurde - aus genau dem Grund, daß Großbritannien weder Wettbewerb noch unabhängige Entwicklung duldete. Neuengland hingegen war in der Lage, dem Weg des Mutterlandes zu folgen, indem es billigere britische Textilien durch sehr hohe Zölle abwehrte - ganz so wie Großbritannien es mit Indien gemacht hatte. Ohne solche Maßnahmen wäre die Hälfte der aufstrebenden Textilindustrie Neuenglands zerstört worden, mit all den offensichtlichen Konsequenzen für viele andere Industrien, wie die einzige Untersuchung des Themas durch einen Wirtschaftshistoriker schließt (Bils 1984). Es ist merkwürdig, daß die zentrale Frage der amerikanischen Wirtschaftsgeschichte praktisch nicht auf der Tagesordnung steht und offenbar als »politisch inkorrekt« angesehen wird.

Gewiß, Großbritannien wandte sich schließlich dem liberalen Internationalismus zu - im Jahr 1846, nachdem 150 Jahre Protektionismus, Gewalt und die Errichtung eines starken und effizienten Staates ihm eine Industrialisie- 
rung gesichert hatten, die pro Kopf mehr als zweimal so stark war, wie bei jedem anderen Konkurrenten, so daß das »Spielfeld « jetzt ziemlich sicher aussah. 1846 exportierte Indien überhaupt keine Baumwollgüter mehr und mußte Tuch aus England einführen - mehr als viermal so viel wie 10 Jahre vorher. England war endlich führend in der Textilherstellung geworden, nachdem es die Ent-Industrialisierung Indiens mit Gewalt verwirklicht hatte. »Es fällt doch auf«, bemerkt Mukerjee, »daß die englischen Ökonomen und Staatsmänner erst zu Anhängern der Lehre der freien Marktwirtschaft als dem sichersten Weg zum Reichtum der Völker wurden, nachdem sich die Baumwollindustrie von Lancashire entwickelt hatte - mit Hilfe von Zöllen und Verboten gegenüber französischen Waren, irischen Wollsachen und indischen Seide- und Baumwollimporten«. Die Maßnahmen Britanniens waren extrem und gingen über hohe Schutzzölle hinaus. Die Behauptung der Ideologen, daß Adam Smith $\gg$ England von den Vorzügen des freien internationalen Handels überzeugt habe« (so George Stigler, Nobelpreisträger der Wirtschaftswissenschaften von der Universität Chicago) hält den einfachsten empirischen Tatsachen nicht stand.

1846 wandte sich Großbritannien endgültig dem liberalen Internationalismus zu - aber nicht ohne deutliche Vorbehalte. So gingen weiterhin $40 \%$ der britischen Textilien in die indische Kolonie, und ziemlich dasselbe traf auf die britischen Exporte generell zu. Im letzen Abschnitt des 19. Jahrhunderts wurde britischer Stahl durch sehr hohe Zölle vom US-Markt ferngehalten, wodurch die USA ihre eigene Stahlindustrie entwickeln konnten; der bekannte Pazifist Andrew Carnegie war in der Lage, das erste Milliardenunternehmen der Welt aufzubauen; dank der hohen Zölle, der Verträge mit der Marine und der Hilfe der Staatsgewalt bei der Niederhaltung der Arbeiterorganisationen und der Errichtung einer regelrechten Tyrannei über die Industriestädte. Aber Indien und andere Kolonien waren immer noch verfügbar, wie auch später, als der britische Stahl durch niedrigere Preise von den internationalen Märkten verdrängt wurde. Indien wiederum ist ein besonders interessanter Fall; es produzierte gegen Ende des 18. Jahrhunderts so viel Stahl wie ganz Europa zusammengenommen britische Ingenieure studierten noch 1820 die indischen Techniken bei der Stahlproduktion, »um den britischen Stahlproduzenten zu helfen, die Technologielücke zu Indien zu schließen«, so ein Harvard-Militärhistoriker, und Bombay produzierte wettbewerbsfähige Lokomotiven, als der Eisenbahnboom einsetzte. Aber die »Doktrin der real existierenden freien Marktwirtschaft« zerstörte diese Bereiche der indischen Industrie, wie sie die indische Textilindustrie, den fortgeschrittenen Schiffsbau und die anderen Branchen zerstörte, die das Land vor der britischen Übernahme zum führenden Produktionszentrum gemacht hatten. Im Gegensatz dazu konnten die USA und Japan das britische Modell der radikalen Verletzung der 
Marktgesetze adaptieren. Und als der japanische Wettbewerb zu stark wurde, drehte England den Spieß einfach um: Das Empire verschloß sich den japanischen Exporten, was nicht unbeträchtliche Auswirkungen auf die Entstehung des Zweiten Weltkrieges im Pazifik hatte. Die indischen Produzenten verlangten zur selben Zeit nach Protektion - aber gegen England, nicht gegen Japan. Doch unter der Doktrin der real existierenden freien Marktwirtschaft hatte es kein Glück (Rosen 1995, Mukerjee 1967).

Ein Jahrhundert nachdem England zum liberalen Internationalismus zurückgekehrt war - zeitweise und mit Einschränkungen - schlugen die USA aus ähnlichen Gründen denselben Kurs ein. 1945, nach 150 Jahren des extremen Protektionismus, der Gewalt und der Schaffung eines Staates, der wirksam in die Entwicklung eingriff, waren die Vereinigten Staaten das bei weitem reichste und mächtigste Land der Welt geworden und erkannte nun plötzlich, wie vorher England, die Meriten des liberalen Internationalismus. Doch wiederum mit entscheidenden Einschränkungen. Eine war, daß Washington, wie Großbritannien, seine Macht nutzte, um unabhängige Entwicklungen anderswo zu blockieren. Lateinamerika wurde eine »ergänzende«, aber keine »wettbewerbsfähige« Entwicklung erlaubt, eine harte Bedingung für dieses "Versuchsgelände für wissenschaftliche Methoden der Entwicklung« in Übereinstimmung mit dem amerikanischen Kapitalismus. Hilfen für das erst vor kurzem unabhängig gewordene Ägypten und (auf verschlungenen Wegen) für Indien waren an ähnliche Bedingungen geknüpft. Versuche, diese Regeln zu verletzen, haben oft extreme Gewalt provoziert; unter dem Vorwand des Kalten Krieges, wenn es gerade paßte, ansonsten unter anderen Vorwänden.

Ein weiterer Vorbehalt war (und ist) im Inland zu finden. Eine entscheidende Komponente der Freihandelstheorie ist die Ablehnung öffentlicher Subventionen. Doch die amerikanische Geschäftswelt und führende Wirtschaftswissenschaftler befürchteten eine Rückkehr zur Großen Depression, als die überhitzte Konsumnachfrage aus dem Krieg erschöpft war und die Wirtschaftsführer sich bewußt wurden, daß die entwickelte Industrie »nicht in einer rein wettbewerbsbestimmten, nicht-subventionierten Wirtschaft 'freier Unternehmer' existieren kann« und daß »die Regierung ihre einzige Rettung ist«. Die Wirtschaftsführer setzten rasch auf das Pentagon-System als optimales Mittel, um der Öffentlichkeit die Kosten aufzuerlegen, während die Gewinne privatisiert werden - aus sehr verständlichen Gründen. Man war sich klar, daß Sozialausgaben dieselbe stimulierende Rolle spielen konnten, aber sie sind keine direkten Subventionen für den hochtechnisierten Unternehmenssektor, und es gibt auch einige unerwünschte Folgen. Sozialausgaben haben demokratisierende Wirkung: Die Leute haben eine Meinung darüber, wo ein Krankenhaus oder eine Schule stehen sollte, aber sie haben keine Meinung über ein Luftverteidigungssytem, das die Grund- 
lagen für die marktfähigen Computer schuf. Und Sozialausgaben haben meist eine umverteilende Wirkung. Militärausgaben haben keinen dieser Fehler, und sie sind leicht zu verkaufen, zumindest solange die demokratischen Formen durch Täuschung und Manipulation ihrer Substanz beraubt werden können. Stuart Symington, Luftwaffenminister unter Truman, hat im Januar 1948 den Tatbestand unverblümt formuliert: »Das Wort, das man benutzen mußte, war nicht 'Subvention'; das Wort war 'Sicherheit'.« Als Industrievertreter in Washington forderte er regelmäßig Beschaffungsfonds im Militäretat, um »die Anforderungen der Flugzeugindustrie zu erfüllen«, wie er es formulierte. Eine Folge ist, daß »Zivilflugzeuge« jetzt der Hauptexportartikel des Landes sind und daß die riesige Reise- und Tourismusindustrie, die auf Flugzeugen beruht, für große Gewinne und eine ausgesprochen vorteilhafte Handelsbilanz bei den Dienstleistungen sorgt. Dasselbe Muster herrscht in der Computerindustrie vor, generell bei elektronischen Geräten, der Metallurgie, Biotechnologie, Telekommunikation und Informatik, tatsächlich in fast jedem dynamischen Sektor der Wirtschaft.

Es ist schwer, das Gesicht nicht zu verziehen, wenn »missionarische Anhänger der freien Marktwirtschaft« die »konservativen Regierungen in den USA und anderswo « für ihre marktwirtschaftliche Politik preisen und das »angelsächsische laisser-faire « (Kelsey 1995: 10, 17, 19) bewundern. Solche Posen mögen in den akademischen Institutionen durchgehen, aber sie würden auf den Korridoren der Macht, der Wirtschaft oder des Staates, einfach nur Gelächter hervorrufen.

Die Geschichte geht weiter bis in die Gegenwart. Es war nicht notwendig, die »Doktrin der real existierenden freien Marktwirtschaft« den ReaganAnhängern zu erklären, die ja Meister in der Kunst waren, die Segnungen des Marktes gegenüber den Armen zu Hause und den abhängigen Gebieten im Ausland zu preisen, während sie gegenüber der Geschäftswelt stolz damit prahlten, daß Reagan »der US-Industrie mehr Schutz vor Importen gewährt hatte als irgendeiner seiner Vorgänger in über einem halben Jahrhundert« (dies sagte Finanzminister James Baker, der viel zu bescheiden war; in Wirklichkeit war es mehr als bei allen Vorgängern zusammengenommen, denn die Reagan-Regierung verdoppelte die Importbeschränkungen). Inzwischen hatte die Regierung den Transfer von öffentlichen Mitteln zu Privatpersonen beschleunigt, besonders durch das Pentagonsystem. Ohne diese extremen Verletzungen marktwirtschaftlicher Prinzipien wäre es zweifelhaft, ob zentrale Sektoren der Industrie wie Stahl, Fahrzeugbau, Werkzeugmaschinen- oder Halbleiterproduktion den japanischen Wettbewerb überlebt hätten, oder ob aufstrebende Industrien in der Lage gewesen wären, voranzukommen - mit Auswirkungen auf die ganze übrige Wirtschaft. Diese Erfahrung verdeutlicht einmal mehr, daß »die herkömmliche 
Weisheit voller Löcher« ist, wie Alan Tonelson in einer Bilanz der Marktinterventionen der Regierung Reagan in Foreign Affairs (Juli/Aug. 1994) herausstellte. Aber die herkömmliche Weisheit behält ihre Vorteile als ideologische Waffe zur Disziplinierung der Wehrlosen.

Es ist auch nicht notwendig, die Doktrinen dem Führer der heutigen »konservativen Revolution « in Washington, Newt Gingrich, zu erklären, der mit strenger Miene siebenjährige Kinder über das Übel der Abhängigkeit von der Wohlfahrt belehrt, während er den Nationalpreis für die Verteilung von Bundessubventionen an seine reiche Wählerschaft gewinnt dank solcher Paradebeispiele für freies Unternehmertum wie etwa Lockheed, dem größten Arbeitgeber seines Distrikts. Auch der »Heritage Foundation« muß man es nicht erklären, die die Haushaltsentwürfe der »Konservativen « im Kongreß fabriziert und deshalb eine Steigerung des Pentagon-Budgets verlangte - und über die von Clinton vorgesehene hinaus auch erhielt. Sichergestellt werden soll die »Basis der Verteidigungsindustrie«, geschützt werden soll sie vom »fürsorglichen« Staat, und ihren NutznieBern soll sie zweischneidige Technologien an die Hand geben, die ihnen die Herrschaft über die kommerziellen Märkte ermöglichen. Als Clinton den Verteidigungsetat erhöhte, der sehr schnell durch die »Libertären « des Kongresses aufgestockt wurde, war das seine direkte Antwort auf das »breite Wählermandat für den Konservativismus« im November 1994 und wurde von einem beeindruckenden Sechstel der Bevölkerung unterstützt.

Doch alle verstehen sehr gut, daß Demokratie eine Plage ist, die so lange wie möglich ignoriert werden muß, und daß freies Unternehmertum bedeutet, daß die Öffentlichkeit für die Kosten, unter welcher Verkleidung auch immer, aufkommt und die Risiken trägt, wenn die Dinge falsch laufen, während die Gewinne privatisiert werden. Und zur Verwirklichung dieser Ziele müssen die Entscheidungen so weit wie möglich von der öffentlichen Arena weg auf nicht haftbare, private Tyranneien übertragen und in Verträge »eingeschlossen« werden, die die potentielle Bedrohung durch die Demokratie untergraben.

Die neuseeländische Gesetzeskommission mußte erkennen, daß ein entscheidender Bestandteil der internationalen Handelsverträge darin besteht, die »Macht des neuseeländischen Parlaments substantiell zu beschränken« (Kelsey 1995: 104). Das ist auch zu einem großen Teil ihre Funktion. In den USA ist es nicht länger möglich, weiterhin die euphorischen Prognosen über die Vorteile der NAFTA zu verbreiten, so daß die Bildungseliten stillschweigend eingestehen, daß die Befürworter der NAFTA von Anfang gelogen haben. Die Clinton-Regierung »vergaß, daß der Sinn der NAFTA nicht darin bestand, den Handel zu fördern, sondern die Wirtschaftsreformen Mexikos zu festigen« erklärt voller Hochmut der Newsweek-Korrespondent Marc Levinson in Foreign Affairs (März/April 1996), ohne aller- 
dings hinzufügen, daß genau das Gegenteil lautstark gefordert wurde, um die Annahme der NAFTA zu erreichen, während die Kritiker, die die »dahinter stehende Absicht« betonten, von der Debatte wirksam ausgeschlossen wurden.

Wir können heute zugeben, daß das Hauptziel der NAFTA nicht darin lag, die als Geheimtip gehandelten Wunder beim »Handel « und bei den »Jobs« zu erreichen - das ist immer eine Illusion gewesen - sondern darin, sicherzustellen, daß Mexiko bei den »Reformen« blieb, die es zu einem »Wirtschaftswunder« gemacht hatten - für US-Investoren und die mexikanischen Eliten. So wurde die Gefahr gebannt, die bei einem Workshop zur StrategieEntwicklung für Lateinamerika im September 1990 im Pentagon aufgedeckt wurde: Daß »eine 'demokratische Öffnung' in Mexiko die besonderen Beziehungen in Frage stellen könnte, wenn eine Regierung ins Amt käme, die aus wirtschaftlichen oder nationalistischen Beweggründen daran interessiert wäre, die USA herauszufordern. «Trotz einer reichen Auswahl an verfügbaren Mitteln, um die Bedrohung durch die Demokratie abzuwehren, können die Mächtigen nicht ausschließen, daß die Seuche irgendwo ausbricht.

Natürlich sind die USA in ihren Vorstellungen vom »freien Handel« nicht alleine, wenn ihre Ideologen auch den zynischen Chor anführen. Laut Entwicklungsbericht der UNO von 1992 ist die Verdopplung des Abstandes zwischen armen und reichen Ländern seit 1960 grundsätzlich den protektionistischen Maßnahmen der Reichen zuzuschreiben. Die Praktiken dauerten während der Uruguay-Runde an, wie der UNDP-Bericht 1994 bemerkt und weiter folgert, daß »die Industrieländer durch die Verletzung des Prinzips des freien Handels die Entwicklungsländer schätzungsweise 50 Milliarden Dollar im Jahr kosten - fast so viel wie die gesamte Entwicklungshilfe«, von der ein großer Teil lediglich eine öffentlich subventionierte Exportförderung ist (Toussaint/Drucker 1995).

Um es auf andere Art zu illustrieren: Eine neuere Studie über die 100 größten multinationalen Konzerne in der Rangliste von Fortune zeigt, daß praktisch alle mehr oder weniger erfolgreich versucht haben, von der Industrie- und/oder Handelspolitik (ihrer eigenen Regierung) zu profitieren, und »mindestens 20 ... hätten nicht als unabhängige Unternehmen überlebt, wenn sie nicht irgendwie von ihren Regierungen gerettet worden wären.« Eine von Gingrichs bevorzugten »Cash cows «, Lockheed, wurde durch einen 2-Milliarden-Dollar-Kredit der Nixon-Regierung vor dem Bankrott gerettet. Wiederum betritt Neuseeland kein Neuland, wenn seine Libertären "Electricorp « in dem Augenblick aus der Patsche helfen, da die Firma in Schwierigkeiten gerät (vgl. dazu W. Ruigrok Financial Times 5.Jan. 1996, McQuaid 1994).

Über diese Angelegenheit gäbe es noch eine Menge mehr zu sagen, doch einige Schlußfolgerungen scheinen schon ziemlich klar zu sein: Wie in den 
Tagen von Smith und später Ricardo werden die bewährten Lehren aus Macht- und Profitgründen sorgfältig ausgearbeitet und angewandt. Es ist nichts Neues, wenn das »Neuseeland-Experiment« die Form eines »Sozialismus für die Reichen « annimmt. Das gehört zum internationalen »Triumph des Marktes $«$, der auf einem System des globalen Unternehmensmerkantilismus beruht, in dem »Handel « substantiell aus zentral geleiteten firmeninternen Transaktionen und Interaktionen zwischen riesigen Institutionen besteht - totalitär in der Substanz und dazu bestimmt, den demokratischen Entscheidungsprozeß zu unterlaufen und die Herren vor den Gesetzen des Marktes zu schützen; ein System, in dem »oligopolistischer Wettbewerb und strategische Interaktionen unter Firmen und Regierungen anstelle unsichtbarer Marktkräfte die heutigen Wettbewerbsvorteile und die internationale Arbeitsteilung in den Hochtechnologie-Ländern bestimmen « (OECD 1992). Es sind die Armen und Wehrlosen, die in der strengen Lehre von den Gesetzen des Marktes unterrichtet werden müssen.

Seit den Anfängen der Industriellen Revolution hat es wiederholt Bemühungen gegeben, innerhalb der industrialisierten Gesellschaften selbst jene Sorte von »Experimenten « durchzuführen, die anderen Ländern aufgezwungen wurden - aber der Erfolg war nur mäßig. Den ersten Versuch gab es im England des frühen 19. Jahrhunderts, als die Lehren des »Neoliberalismus« als Instrumente des Klassenkampfs geschmiedet wurden: speziell die Lehre, daß man durch das Bemühen, den Armen zu helfen, ihnen nur schadet, und daß die Menschen kein anderes Recht haben als das, was sie auf dem Arbeitsmarkt gewinnen können; ganz im Gegensatz zu den mißverstandenen Annahmen einer vorkapitalistischen Gesellschaft, die ein törichtes »Recht auf Leben« hochhielt. Wer unter dem rauhen Marktregime nicht überleben kann, mag in das Arbeitshaus-Gefängnis gehen, oder besser noch, sonstwohin - in jener Zeit nicht unmöglich, da Nordamerika und Teile des Pazifiks gerade von der Eingeborenenplage befreit wurden. Das sind wirkliche Naturgesetze, wie Ricardo und andere allen Ernstes erklärten, genau so gewiß wie das Gravitationsgesetz.

Mit dem Triumph des richtigen Denkens im Dienste der britischen Industrie- und Finanzinteressen wurde das Volk »auf den Weg eines utopischen Experiments gezwungen «, schreibt Karl Polanyi (1944) in seinem klassischen Werk; es handelte sich um den »rücksichtslosesten Akt der Sozialreform « in der ganzen Geschichte, der »eine große Zahl von Menschenleben zerstörte «. Aber es entstand ein Problem. Die dummen Massen, die unfähig waren, die zwingende Logik der Wissenschaft zu verstehen, fingen an, die Schlußfolgerung zu ziehen, daß wenn sie kein Recht zum Leben hätten, niemand anderes das Recht zum Herrschen hätte. Die britische Armee mußte Aufstände und Unruhen niederschlagen, und bald nahm eine noch größere Bedrohung Gestalt an: »Fabrikgesetze und Sozialgesetze sowie ei- 
ne politische und industrielle Arbeiterbewegung erblickten das Licht der Welt ... um den ganz neuen Gefahren der Marktgesetze zu begegnen." Chartisten und Sozialisten verbreiteten sogar noch größeren Schrecken. Die glücklicherweise geschmeidige Wissenschaft nahm neue Formen an, als sich in Reaktion auf die unkontrollierbaren Kräfte des Volkes die Ansichten der Eliten änderten und man erkannte, daß das »Recht auf Leben « in einer Art von Gesellschaftsvertrag erhalten werden mußte.

Auch diese Geschichte hat sich über die Jahre hinweg wiederholt, in den USA und in anderen Industriegesellschaften. Heute ist der Gesellschaftsvertrag, erstritten von unten, wieder einmal Gegenstand von Angriffen, besonders in den angloamerikanischen Gesellschaften. Das ist ein Aspekt dessen, was die Wirtschaftspresse die »klare Unterwerfung der Arbeit durch das Kapital in den letzten 15 Jahren« nennt (J. Liscio, Barron's, 15.Apr. 1996). Die neuen Experimente werden wie immer von zuversichtlichen Aufrufen begleitet, die alle den Respekt verdienen, den sie sich in der Vergangenheit erworben haben.

Führt man sich dies vor Augen, muß man vorsichtig und mit aller Sorgfalt die »neoliberale Wirtschaft und Philosophie evaluieren, welche die intellektuelle Diskussion, ausgehend vor allem von den USA, beherrschen« (Kelsey 1995: 17) - mit besonderer Berücksichtigung des rationalen Gehalts der Argumentationen und der Lektionen der vergangenen und gegenwärtigen Geschichte, wozu nicht zuletzt der Zynismus der intellektuellen Debatte zur Verschleierung der $»$ Doktrin der real existierenden freien Marktwirtschaft« gehört. Es macht wenig Sinn danach zu fragen, was »richtig « für die USA ist (oder für Indien, Neuseeland etc), als ob diese Länder Gebilde mit gleichen Interessen und Werten wären. Auf dem Feld praktischer Entscheidungen ist das selten der Fall. Und was für die Menschen in den USA richtig sein mag, mit all ihren beispiellosen Vorteilen, könnte für andere sehr wohl falsch sein, die viel begrenztere Wahlmöglichkeiten haben, Wahlen, die sie auf dem Hintergrund besonderer historischer und soziokultureller Kontingenzen treffen müssen. Wir können aber mit einigem Grund annehmen, daß, was richtig für das amerikanische (oder das indische, neuseeländische) Volk ist, nur im unwahrscheinlichsten Fall mit den Vorstellungen der »führenden Architekten der Politik« übereinstimmen wird - aus ziemlich den gleichen Gründen, die Adam Smith so gut verstand.

Aus dem Englischen übersetzt von Günter Barwig

\section{Literatur}

Bairoch, P. (1993): Economics and World History, Chicago.

Bayly, C. A. (1988): The New Cambridge History of India, Cambridge.

Bils, M. (1984): Journal of Economic History, No. 4. 
Chandra, B. (1971): Modern India, Delhi.

Chomsky, N. (1993): Year 501, Boston.

Cullaher, N. (1996): The U.S. and Taiwanese Industrial Policy, in: Diplomatic History, vol. 20, no 1.

Eckes, A. (1995): Opening America's Market: U.S. Foreign Trade Policy since 1776, Chapel Hill \& London.

Kelsey, J. (1995): The New Zealand Experiment. A World Model for Structural Adjustment, Auckland.

Komya, R., et aI. (1988): Industry Policy of Japan, Tokyo.

Krugman, P. (1995): Cycles of conventional wisdom on economic development«, in: International Affairs, vol. 71, no 4.

Levinson, M. (1996): Newsweek, FA March/April.

McQuaid, K. (1994): Uneasy Partners, Baltimore, London.

Mukerjee, R. (1967): The Economic History of India: 1600-1800, Allahabad.

Polanyi, Karl (1944): The Great Transformation, Frankfurt/M 1978.

Rosen, S. (1995): Military Effectiveness, in: International Security, vol. 19, no 4.

Rothermund, D. (1993): An Economic History of India, London.

Stigler, G. (1976): Introduction, in: Smith, A. Wealth of Nations, Bicentennial edition, University of Chicago.

Toussaint, E.; Drucker, P. (eds.) (1995): IMF/World Bank/WTO, Notebooks for Study and Research 24/5, Amsterdam.

Wei-ching Wang, V. (1995-96): Developing the Information Industry in Taiwan, in: Pacific Affairs, vol. 68 , no. 4 .

\section{Ungleichheit als Projekt. Globalisierung - Standort - Neoliberalismus.}

Fachübergreifende Tagung am 28. und 29.11.1997 in Marburg.

Veranstaltet vom Bund demokratischer Wissenschaftlerinnen und Wissenschaftler, von der Arbeitsgruppe Alternative Wirtschaftspolitik (Memogruppe) und dem Institut für Politikwissenschaft der Universität Marburg.

Mit Elmar Altvater, Detlev Hensche, Jürgen Trittin, Ingrid Kurz-Scherf, Jörg Huffschmid, Wolfgang Fach, Clemens Knobloch, Frank Deppe u.v.m.

\section{Kontaktadresse:}

Regina Stötzel/Dr. Rainer Rilling, c/o BdWi, Postfach 543, 35017 Marburg Telefon 06421/21395, Telefax 06421/24654

Mail: rillingr@mailer.uni-marburg.de

Infos im Internet:

http://staff-www.uni-marburg.de/ rillingr/bdweb/ungleichheit.htm 